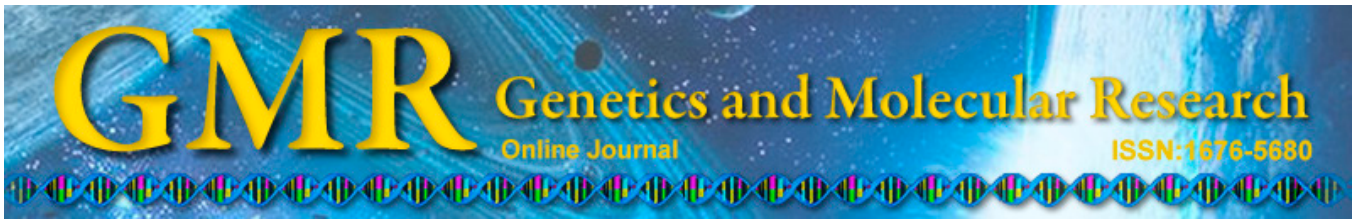

\title{
Association between serum angiopoietin-2 concentration and clinicopathological parameters in patients with colorectal cancer
}

\author{
Z.Q. Wang ${ }^{1 *}$, X.L. Sun ${ }^{2 *}$, H.L. Su ${ }^{3}$, X.F. Liu ${ }^{4}$, Y.J. Xuan ${ }^{1}$ and S.Q. Yu ${ }^{5}$ \\ ${ }^{1}$ Department of Anorectal Surgery, Yantai Yuhuangding Hospital, \\ Yantai, Shandong, China \\ ${ }^{2}$ Department of Laboratory Medicine, Yantai Yuhuangding Hospital, \\ Yantai, Shandong, China \\ ${ }^{3}$ Department of General Surgery, Yantai Yuhuangding Hospital, \\ Yantai, Shandong, China \\ ${ }^{4}$ Department of Hepatobiliary Surgery, Yantai Yuhuangding Hospital, \\ Yantai, Shandong, China \\ ${ }^{5}$ Department of Urology, Yantai Yuhuangding Hospital, Yantai, \\ Shandong, China \\ *These authors contributed equally to this study. \\ Corresponding author: X.L. Sun \\ E-mail: dr_sunxiaoli@126.com
}

Genet. Mol. Res. 14 (4): 15547-15552 (2015)

Received July 23, 2014

Accepted March 26, 2015

Published December 1, 2015

DOI http://dx.doi.org/10.4238/2015.December.1.5

\begin{abstract}
We examined the expression of angiopoietin-2 in serum samples from patients diagnosed with colorectal cancer and healthy volunteers and investigated the feasibility of using angiopoietin-2 as a potential diagnostic colorectal cancer biomarker. Enzymelinked immunosorbent assays were used to measure the levels of angiopoietin-2 in patients with colorectal cancer and healthy control subjects. Correlations between serum angiopoietin-2 levels and clinicopathological factors were investigated. A receiver operating characteristic curve was used to predict cut-off values of the markers.
\end{abstract}


Serum concentrations of angiopoietin-2 were significantly higher in patients with colorectal cancer than in controls $(2896 \pm 1273$ vs 1554 $\pm 991 \mathrm{pg} / \mathrm{mL}, \mathrm{P}=0.004)$. Serum angiopoietin-2 expression levels were significantly positively correlated with TNM stage $(\mathrm{P}=0.003)$, lymph node involvement $(\mathrm{P}=0.04)$, and distant metastases $(\mathrm{P}=0.005)$. Receiver operating characteristic curve analysis revealed that serum level of angiopoietin-2 was a potential biomarker for differentiating colorectal cancer patients from controls and had a receiver operating characteristic area under the curve of 0.859 (95\% confidence interval $=0.740-0.978)$. At a cut-off value of $2710 \mathrm{pg} / \mathrm{mL}$, the sensitivity was $79.3 \%$ and the specificity was $82.4 \%$. Our results suggest that angiopoietin- 2 can be used as a diagnostic biomarker for colorectal cancer in clinical practice. Additional studies are needed to clarify the detailed mechanism of angiopoietin-2 in the carcinogenesis and metastasis of colorectal cancer.

Key words: Angiopoietin-2; Colorectal cancer; Diagnostic biomarker

\section{INTRODUCTION}

Colorectal cancer is a leading cause of cancer mortality worldwide, causing approximately 400,000 deaths per year (Jemal et al., 2008). With economic development and dietary pattern changes, the incidence of colorectal cancer has increased rapidly in China. Currently, colorectal cancer is the fourth most common malignant tumor and the third leading cause of cancer death in China (Long et al., 2010). Metastasis is the major cause of colorectal cancer mortality, and more than one third of patients with colorectal cancer will ultimately develop metastatic disease (Center et al., 2009). Therefore, it is urgent to identify more specific and sensitive biomarkers for the early diagnosis and prognosis prediction of colorectal cancer.

Angiogenesis is an essential process in cancer growth, maintenance, and metastasis (Folkman et al., 1989). Tumor vessel density, which represents angiogenesis, is related to metastasis and has prognostic value in various malignant tumors, including colorectal cancer. Angiogenesis is controlled by the interplay between numerous positive and negative factors (Yancopoulos et al., 2000). Angiopoietin-2 is a ligand of the tyrosine kinase receptor Tie 2 and integrin receptors (Takanami, 2004). It functions as an autocrine controller of endothelial cells in a context-dependent manner, promoting either blood vessel growth or regression depending on the levels of other growth factors such as vascular endothelial growth factor (VEGF)-A (Holash et al., 1999; Zhang et al., 2003). Circulating angiopoietin-2 level has been associated with tumor angiogenesis in several cancers (Fawzy et al., 2012; Li et al., 2013a,b; Pappa et al., 2013; Chen et al., 2014; Kawaguchi et al., 2014). Engin et al. (2012) found that the concentration of angiopoietin-2 (median $3188.0 \mathrm{pg} / \mathrm{mL}$, min: $1070.5 \mathrm{pg} / \mathrm{mL}$, max: $5765.5 \mathrm{pg} / \mathrm{mL}$ ) was significantly higher in patients with colon cancer, while concentrations of angiopoietin-1 were not statistically different between groups (Engin et al., 2012). However, they did not investigate the diagnostic value of angiopoietin-2 in colorectal cancer.

In the present study, we examined the expression of angiopoietin-2 in serum samples from patients diagnosed with colorectal cancer and in healthy volunteers. Furthermore, we investigated the feasibility of using angiopoietin- 2 as a diagnostic colorectal cancer biomarker. 


\section{MATERIAL AND METHODS}

\section{Subjects}

In the present study, 98 patients newly diagnosed with colorectal cancer were enrolled from the Department of Anorectal Surgery in Yantai Yuhuangding Hospital between May 2011 and July 2012. Tumors were staged according to the 7th edition of the tumor, node, and metastasis (TNM) classification system of the UICC. Blood samples were collected prior to surgical treatment. Patients who had received preoperative radiotherapy or chemotherapy were excluded. For the control group, we selected 90 healthy volunteers who did not have a history of malignant disease. Written informed consent was obtained from all participants after an explanation of the study, and the study was approved by the Ethics Committee of Yantai Yuhuangding Hospital.

\section{Angiopoietin-2 measurement}

Serum samples were taken preoperatively at the time of diagnosis. First, $10 \mathrm{~mL}$ blood was drawn into serum tubes and centrifuged at $2200 \mathrm{~g} / \mathrm{min}$ for $10 \mathrm{~min}$. Serum was harvested, aliquoted, and stored at $-70^{\circ} \mathrm{C}$ until use. Enzyme-linked immunosorbent assays were used to measure the levels of angiopoietin-2 according to manufacturer instructions (Quantikine; R\&D Systems, Minneapolis, MN, USA). All samples were examined in duplicate, and the mean values were used for statistical analysis. Measurements were conducted in a blinded manner.

\section{Statistical methods}

Data were analyzed using SPSS statistical package version 15 (SPSS Inc., Chicago, IL, USA). Chi-square test (Fisher exact test) was used to examine the relationship between qualitative variables. For quantitative data, comparison between 2 groups was conducted using the Mann-Whitney test. The Spearman-rho method was used to test the correlation between numerical variables. The receiver operating characteristic (ROC) curve was used to predict cut-off values of the markers. Data are reported as means \pm standard deviation. A P value $<$ 0.05 was considered to be significant.

\section{RESULTS}

\section{Expression level of angiopoietin-2 in serum samples and relationship between expression and clinicopathological features}

Serum concentrations of angiopoietin-2 were significantly higher in patients with colorectal cancer than in controls $(2896 \pm 1273$ vs $1554 \pm 991 \mathrm{pg} / \mathrm{mL}, \mathrm{P}=0.004)$. As shown in Table 1, serum angiopoietin-2 expression levels were significantly positively correlated with TNM stage $(\mathrm{P}=0.003)$, lymph node involvement $(\mathrm{P}=0.04)$, and distant metastases $(\mathrm{P}=$ 0.005). However, no significant correlation was observed between serum angiopoietin-2 level and other clinicopathologic parameters tested, including age $(\mathrm{P}=0.41)$, gender $(\mathrm{P}=0.53)$, tumor location $(\mathrm{P}=0.73)$, and tumor size $(\mathrm{P}=0.09)$. 
Table 1. The correlation of angiopoietin-2 expression and clinicopathological features in colorectal cancer patients.

\begin{tabular}{|c|c|c|c|}
\hline Variable & No. of cases & Serum angiopoietin- 2 expression, $\mathrm{pg} / \mathrm{mL}($ mean $\pm \mathrm{SD})$ & $P$ value \\
\hline \multicolumn{4}{|l|}{ Gender } \\
\hline Male & 50 & $2933 \pm 1278$ & \multirow{2}{*}{0.53} \\
\hline Female & 48 & $2831 \pm 1109$ & \\
\hline \multicolumn{4}{|l|}{ Age (years) } \\
\hline$<60$ & 37 & $2781 \pm 1022$ & \multirow[t]{2}{*}{0.41} \\
\hline$\geq 60$ & 61 & $2971 \pm 1255$ & \\
\hline \multicolumn{4}{|c|}{ Tumor location } \\
\hline Colon & 53 & $2866 \pm 1271$ & \multirow[t]{2}{*}{0.73} \\
\hline Rectum & 45 & $2901 \pm 1261$ & \\
\hline \multicolumn{4}{|l|}{ Tumor size } \\
\hline$<5 \mathrm{~cm}$ & 58 & $2533 \pm 1198$ & \multirow[t]{2}{*}{0.09} \\
\hline$\geq 5 \mathrm{~cm}$ & 40 & $3109 \pm 1201$ & \\
\hline \multicolumn{4}{|c|}{ Lymph node metastasis } \\
\hline Negative & 39 & $2534 \pm 1309$ & \multirow[t]{2}{*}{0.04} \\
\hline Positive & 59 & $3091 \pm 1101$ & \\
\hline \multicolumn{4}{|c|}{ Distant metastasis } \\
\hline Negative & 81 & $2411 \pm 1261$ & \multirow[t]{2}{*}{0.005} \\
\hline Positive & 17 & $3905 \pm 679$ & \\
\hline \multicolumn{4}{|l|}{ TNM stage } \\
\hline & 47 & $1921 \pm 997$ & \multirow{2}{*}{0.003} \\
\hline III/IV & 51 & $3837 \pm 1289$ & \\
\hline
\end{tabular}

$\mathrm{SD}=$ standard deviation.

\section{Relationship between expression of angiopoietin-2 in serum samples and diagnosis}

Because angiopoietin-2 expression level was significantly elevated in the serum of colorectal cancer patients compared with healthy controls, ROC curve analysis was used to explore the potential of using circulating angiopoietin-2 as a biomarker for colorectal cancer. Angiopoietin- 2 could be used to differentiate colorectal cancer patients from controls, with an area under the ROC curve of 0.859 (95\% confidence interval $=0.740-0.978$; Figure 1$)$. At a cut-off value of $2710 \mathrm{pg} / \mathrm{mL}$, the sensitivity was $79.3 \%$ and the specificity was $82.4 \%$.

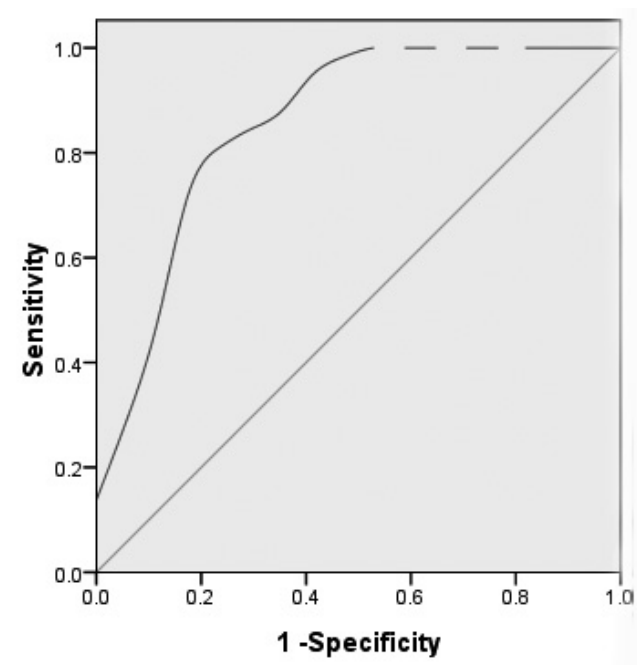

Figure 1. ROC curve for the serum angiopoietin-2 differentiating colorectal cancer and control group. The sensitivity and specificity of angiopoietin-2 were 79.3 and $82.4 \%$, respectively, with $2710 \mathrm{pg} / \mathrm{mL}$ of cut-off value. 


\section{DISCUSSION}

Despite great efforts to improve the prevention and treatment of colorectal cancer, the morbidity and mortality of this disease remains high (Jemal et al., 2008). The tumorigenesis of colorectal cancer is a multi-step process mediated by accumulating alterations in cell proliferation ability and a wide range of genetic disorders (Fearon, 2011). Recent studies have focused on identifying new biomarkers and aberrant genetic features in colorectal cancer.

Increasing evidence supports the essential role of the Ang/Tie-2 system in angiogenic regulation (Quartarone et al., 2006). Angiopoietin-2 acts as a destabilizing signal and promotes tumor angiogenesis by priming the vasculature and potentiating the effects of VEGF at the front of active neovascularization (Lobov et al., 2002). Although malignant tumors undergo massive tumor cell loss via central regression and necrosis, the remaining tumor grows via active angiogenesis at the tumor margin. Angiopoietin- 2 has been reported to be a critical regulator of this angiogenic process. VEGF up-regulation along with angiopoietin-2 expression at the tumor periphery is associated with rescuing the surviving tumor and promoting further growth. Specifically, angiopoietin-2 is strongly induced in the peripheral vessels of tumor tissues before VEGF induction.

Because angiopoietin-2 is a secreted protein, the serum level of angiopoietin- 2 and its clinical value have been investigated in several cancers. For example, Sallinen et al. (2010) found that angiopoietin-2 level was significantly elevated in serum samples of patients with ovarian cancer compared with healthy controls $(\mathrm{P}=0.0005)$. In addition, high angiopoietin-2 levels were associated with poor overall and recurrence-free survival of ovarian cancer patients in univariate analysis. This suggests that angiopoietin- 2 may be an additional diagnostic tool for ovarian cancer, and may serve as an angiogenic marker of decreased patient survival (Sallinen et al., 2010). Similarly, Fawzy et al. (2012) found that serum angiopoietin-2 level in patients with lung cancer was higher than that in the control group. Furthermore, serum angiopoietin-2 may be a useful marker for diagnosing non-small cell lung cancer using the enzyme-linked immunosorbent assay method. Engin et al. (2012) found that the concentration of angiopoietin-2 was significantly higher in patients with colon cancer. However, they did not investigate the diagnostic value of angiopoietin-2 in colorectal cancer.

In the present study, we investigated whether angiopoietin-2 was secreted into the serum of patients with colorectal cancer. Our results showed that the levels of serum angiopoietin-2 were significantly increased in patients with colorectal cancer compared with healthy controls. Furthermore, we found that serum angiopoietin-2 expression levels were significantly positively correlated with TNM stage, lymph node involvement, and distant metastases, suggesting that angiopoietin-2 is involved in the carcinogenesis and metastasis of colorectal cancer. However, in the present study, we did not investigate the detailed mechanism of angiopoietin-2 in the carcinogenesis and metastasis of colorectal cancer. The prognostic value of angiopoietin-2 in colorectal cancer should be investigated in future studies.

In conclusion, our results suggest that angiopoietin-2 can be used as a diagnostic biomarker for colorectal cancer in clinical practice. Additional studies are needed to clarify the detailed mechanism of angiopoietin-2 in the carcinogenesis and metastasis of colorectal cancer.

\section{Conflicts of interest}

The authors declare no conflict of interest. 


\section{REFERENCES}

Center MM, Jemal A and Ward E (2009). International trends in colorectal cancer incidence rates. Cancer Epidemiol. Biomarkers Prev. 18: 1688-1694.

Chen HH, Weng BQ, Cheng KJ, Liu HY, et al. (2014). Effect of the vascular endothelial growth factor expression level on angiopoietin-2-mediated nasopharyngeal carcinoma growth. Vasc. Cell 6: 4.

Engin H, Üstündağ Y, Tekin IO, Gökmen A, et al. (2012). Plasma concentrations of angiopoietin-1, angiopoietin-2 and Tie-2 in colon cancer. Eur. Cytokine Netw. 23: 68-71.

Fawzy A, Gaafar R, Kasem F, Ali SS, et al. (2012). Importance of serum levels of angiopoietin-2 and survivin biomarkers in non-small cell lung cancer. J. Egypt Natl. Cancer Inst. 24: 41-45.

Fearon ER (2011). Molecular genetics of colorectal cancer. Annu. Rev. Pathol. 6: 479-507.

Folkman J, Watson K, Ingber D and Hanahan D (1989). Induction of angiogenesis during the transition from hyperplasia to neoplasia. Nature 339: 58-61.

Holash J, Wiegand SJ and Yancopoulos GD (1999). New model of tumor angiogenesis: dynamic balance between vessel regression and growth mediated by angiopoietins and VEGF. Oncogene 18: 5356-5362.

Jemal A, Siegel R, Ward E, Hao Y, et al. (2008). Cancer statistics, 2008. CA Cancer J. Clin. 58: 71-96.

Kawaguchi M, Sugaya M, Suga H, Miyagaki T, et al. (2014). Serum levels of angiopoietin-2, but not angiopoietin-1, are elevated in patients with erythrodermic cutaneous T-cell lymphoma. Acta Derm. Venereol. 94: 9-13.

Li C, Fan J, Song X, Zhang B, et al. (2013a). Expression of angiopoietin-2 and vascular endothelial growth factor receptor-3 correlates with lymphangiogenesis and angiogenesis and affects survival of oral squamous cell carcinoma. PLoS One 8: e75388.

Li C, Sun CJ, Fan JC, Geng N, et al. (2013b). Angiopoietin-2 expression is correlated with angiogenesis and overall survival in oral squamous cell carcinoma. Med. Oncol. 30: 571.

Lobov IB, Brooks PC and Lang RA (2002). Angiopoietin-2 displays VEGF-dependent modulation of capillary structure and endothelial cell survival in vivo. Proc. Natl. Acad. Sci. U. S. A. 99: 11205-11210.

Long N, Moore MA, Chen W, Gao CM, et al. (2010). Cancer epidemiology and control in north-East Asia - past, present and future. Asian Pac. J. Cancer Prev. 11 (Suppl 2): 107-148.

Pappa CA, Tsirakis G, Samiotakis P, Tsigaridaki M, et al. (2013). Serum levels of angiopoietin-2 are associated with the growth of multiple myeloma. Cancer Invest. 31: 385-389.

Quartarone E, Alonci A, Allegra A, Bellomo G, et al. (2006). Differential levels of soluble angiopoietin-2 and Tie-2 in patients with haematological malignancies. Eur. J. Haematol. 77: 480-485.

Sallinen H, Heikura T, Laidinen S, Kosma VM, et al. (2010). Preoperative angiopoietin-2 serum levels: a marker of malignant potential in ovarian neoplasms and poor prognosis in epithelial ovarian cancer. Int. J. Gynecol. Cancer 20: $1498-1505$.

Takanami I (2004). Overexpression of Ang-2 mRNA in non-small cell lung cancer: association with angiogenesis and poor prognosis. Oncol. Rep. 12: 849-853.

Yancopoulos GD, Davis S, Gale NW, Rudge JS, et al. (2000). Vascular-specific growth factors and blood vessel formation. Nature 407: 242-248.

Zhang L, Yang N, Park JW, Katsaros D, et al. (2003). Tumor-derived vascular endothelial growth factor up-regulates angiopoietin-2 in host endothelium and destabilizes host vasculature, supporting angiogenesis in ovarian cancer. Cancer Res. 63: 3403-3412. 